\title{
Discusiones acerca del concepto de sociología jurídica
}

\author{
IVÁN PACHECO ARRIETA* \\ JORGE ENRIQUE CARVAJAL MARTÍNEZ**
}

Fecha de recepción: 16 de mayo de 2005

Fecha de aprobación: 14 de julio de 2005

\section{RESUMEN}

Cada día la sociología jurídica o sociología del derecho adquiere mayor importancia en el campo de la ciencias jurídicas, importancia que radica en las perspectivas de análisis que la sociología jurídica ofrece al estudio de la norma jurídica y su relación con los fenómenos sociales, sin embargo, como disciplina de reciente formación, existe una controversia acerca del concepto y objeto de la sociología del derecho. Desde un enfoque comparativo, el artículo presenta los aportes realizados por académicos de Europa y América relativos al concepto y objeto de la sociología del derecho.

\section{PALABRAS CLAVES}

Sociología jurídica, criminología, interdisciplinariedad

\section{ABSTRACT}

Every day the juridical sociology or sociology of the law gets more importance in the field of law sciences. This importance takes root in the perspectives of analysis that juridical sociology offers to the study of the laws and its relationships with the social phenomena. Nevertheless, as a subjetc of early formation, there is

\footnotetext{
Ex-secretario General de la Universidad Santo Tomás. Abogado, magister en Sociología Jurídica de la Universidad Oñati.

** Profesor de Sociología Jurídica y Coordinador de publicaciones de la Facultad de Derecho de la Universidad Santo Tomás.
} 
a contrversy about the concept an the object of juridical sociology. From a comparative point of view this article presents the contributions of academicians of Europe and America to those object and concept.

\section{KEY WORDS}

Juridical sociology, criminology, interdisciplinary links

\section{Introducción}

La sociología del derecho es una disciplina de reciente formación. La mayoría de los académicos que abordan esta disciplina coinciden en señalar su aparición en la década de 1960 . Alrededor del significado de la sociología jurídica se han desarrollado importantes debates que tienen como finalidad delimitar el sentido y el concepto de esta disciplina. El punto central gira en torno al reconocimiento de la sociología del derecho como una disciplina autónoma, perteneciente al campo de la sociología general, que aporta importantes elementos para la comprensión del fenómeno del derecho y su relación con los fenómenos sociales.

Desde un enfoque comparativo, el presente artículo se introduce en las discusiones sobre el concepto y objeto de la sociología del derecho. Para cumplir con el anterior propósito se realizó una importante pesquisa que recoge los aportes de los principales sociólogos del derecho de Europa, América y Colombia.

Adicionalmente, el texto presenta los aportes que otras disciplinas han realizado a la sociología del derecho. Se trata de una serie de trabajos teóricos e investigaciones relacionada con temas que también son abordados en el derecho y la sociología del derecho. Nos referimos a una extensa producción de la sociología, la economía, la filosofía social y la antropología nacional, que se ha centrado en las problemáticas de violencia política, democracia, organización territorial, narcotráfico, organización de la familia y movimientos sociales.

\section{1. ¿Qué es la sociología jurídica o sociología del derecho?}

Empecemos por decir que en este texto utilizaremos como sinónimos las expresiones sociología jurídica y sociología del derecho. Éste ha sido un debate de alguna manera recurrente. Sin embargo, para la mayoría de los autores la solución siempre ha sido la de declarar su equivalencia. Así, por ejemplo, Jean Carbonnier aborda los elementos que podrían distinguir a la sociología del derecho de la sociología jurídica, para luego descartarlos y usar ambas expresiones como equivalentes en aras de garantizar un campo de estudio lo más amplio posible. Las posiciones descartadas por Carbonnier se resumen señalando que la sociología del derecho analizaría lo que constituye el derecho mismo, es decir, las reglas y las instituciones; mientras que la sociología jurídica "engloba todos los fenómenos de los que en el derecho pueden ser causa, efecto u ocasión. Incluidos los fenómenos de violación, inefectividad o desviación". Así, para este autor, la sociología del derecho o sociología jurídica, puede definirse como "aquella rama de la sociología general que tiene por objeto una variedad de fenómenos sociales: los fenómenos jurídicos o fenómenos de derecho" (Carbonnier, 1982: 15). 
Con anterioridad a Carbonnier, Georges Gurvitch, profesor de la Universidad de Strasburgo en Francia y del New School for Social Research en Inglaterra, presentaba su definición de la sociología del derecho en los siguientes términos: "Es aquella parte de (la) sociología del espíritu humano que estudia la realidad plena del derecho, comenzando por sus expresiones tangibles y externamente observables en las conductas colectivas efectivas (organizaciones cristalizadas, prácticas y tradiciones consuetudinarias o innovaciones de la conducta) y en la base material (la estructura espacial y la densidad demográfica de las instituciones jurídicas)» (Gurvitch, 1945: 68).

De manera amplia puede decirse que el objeto de estudio de la sociología del derecho lo constituyen las relaciones entre derecho y sociedad. Sin embargo, tal enunciado es vago y poco aporta a la hora de distinguir el objeto de estudio de la sociología jurídica del de otras ciencias que pueden tener temas comunes de interés, como lo antropología, la economía y la historia. La dificultad de esta definición radica en que al pretender ser muy particular puede excluir temas y áreas que han sido y prometen ser de su interés, pero al ampliarla se entra en conflicto con el objeto de otras disciplinas, ciencias y especialidades. Esta diferenciación es muy difícil en la práctica y esforzarse por marcar líneas divisorias puede ser un esfuerzo vano, toda vez que lo realmente importante es el resultado final de los estudios y formulaciones teóricas. Por otra parte, cada vez es más difusa la línea que separa los distintos objetos de estudio, así como sus métodos, propósitos y técnicas profesionales que desde otras disciplinas de las ciencias sociales han incursionado en la investigación sociojurídica.
Sin ánimo distinto al de ilustrar acerca de los diversos criterios que con relación al tema existen, presentamos algunas definiciones, junto con nuestros comentarios sobre las mismas. Hemos incluido pensadores de diversas latitudes, continentes y tradiciones jurídicas.

Renato Tréves distingue en su obra tres concepciones diferentes de la sociología del derecho, las cuales corresponden a etapas distintas de su evolución teórica personal. Ellas son:

1. Disciplina que promueve y desarrolla investigaciones empíricas para fines de carácter práctico, conectadas especialmente con la producción y aplicación del derecho (1966).

2. Disciplina que tiene como objeto de estudio las relaciones entre el derecho y la sociedad y que se divide en dos partes, una teórica y otra empírica $(1977,1980)^{1}$.

3. Disciplina que desarrolla dos tipos de investigaciones diferentes, pero conectadas entre sí e incluso complementarias: la determinación del "derecho libre" o derecho producido fuera de los esquemas jurídicos formales, y el estudio de la situación y función del derecho en la sociedad $(1988)^{2}$.

Las dos primeras aproximaciones se centran en el tipo de investigación, dando, la primera, indiscutido papel protagónico a la investigación empírica, lo que obedecía a la necesidad de garantizar un espacio propio de la sociología jurídica tanto ante los sociólogos como ante los juristas en la Italia de la época. La segunda vuelve al enunciado general del estudio de las relaciones entre derecho y so-

1 Treves señala en el primer párrafo de "La sociología del derecho": "La expresión ‘sociología del derecho', así como la expresión sustancialmente equivalente 'sociología jurídica', sirve para designar una disciplina que tiene como objeto el estudio de las relaciones entre el derecho y la sociedad" (Treves: 19).

2 Este resumen aparece en la nota preliminar de Manuel Atienza, pero la fuente primaria es un texto del mismo Treves "Alla ricerca di una definizione della sociologia del diritto", en: Revista trimestrale di diritto e procedura civile, XLI, 3, septiembre de 1987, pp. 773 - 781. 
ciedad, abriendo la posibilidad a los desarrollos teóricos. La última, pasa a ser mucho más específica. Acoge, por un lado, el llamado derecho libre, y por otro, se ocupa de la situación y función del derecho en la sociedad. Esta aproximación es sin duda más amplia y comprensiva, pero parece excluir a la parte micro del mundo judicial.

El abogado español, Ramón Soriano, en su texto "Sociología del derecho", afirma que: "La sociología del derecho puede ser definida con sencillez y amplitud a través de la interconexión de los dos términos de su nomenclatura: la sociología jurídica se ocupa de la influencia de los factores sociales en el derecho y de la incidencia que éste tiene, a su vez, en la sociedad: la mutua interdependencia de lo social y lo jurídico" (Soriano: 1997: 17), definición que complementa afirmando que "la sociología del derecho es hoy en día una ciencia jurídica sustantiva, además de complementaria de las ciencias jurídicas tradicionales, como demuestra su progresiva institucionalización docente e investigadora en diferentes espacios geográfico - culturales. Soriano (1997: 18) apoya esta afirmación otorgando a la sociología jurídica las siguientes siete características: no-paradigmática, autónoma, independiente, interdisciplinar, informativo-normativa, teórico-empírica y omnicompresiva-acumulativa.

Siguiendo la ruta trazada por Carbonnier, el mexicano Jesús Ángel de la Torre, en su texto "Sociología jurídica y uso alternativo del derecho", da la siguiente definición: "La sociología jurídica es una disciplina científica con relación al derecho, al fenómeno jurídico, ése es su objeto formal, es decir, la perspectiva desde la cual se aborda el análisis del derecho... El quehacer científico de la sociología jurídica consiste en entender el fenómeno jurídico como resultado de procesos sociales, interdependiente del efecto que el derecho tiene como regulador de la acción social" (De la Torre, 1997: 40-44). En esta definición existe un fuerte componente del derecho formal y no resulta cla- ro el vínculo con otras formas jurídicas que se encuentran por fuera de los esquemas formales del derecho, reconocido por el llamado "pluralismo jurídico".

Boaventura de Sousa Santos, sociólogo portugués que ha ejercido importante influencia sobre algunos autores colombianos y de América Latina, ubica la sociología jurídica como una rama especializada de la sociología y se refiere también a los conceptos de fenómeno social y fenómeno jurídico (Santos, 1991: 149).

Para el argentino Oscar Correas "la sociología jurídica o sociología del derecho (...) es una disciplina científica que intenta explicar las causas y efectos del derecho" (Correas 1999: 29) (cursivas en el original), entendiendo por derecho "un discurso de carácter prescriptivo, producido por quien detenta el poder, que es reconocido como legítimo, o bien por la mayor parte de la población de un país, o bien por sus fuerzas armadas, y que organiza la violencia, la cual precisamente se legitima por el reconocimiento de ese discurso como derecho" (Correas 1999: 22). Esta definición nos parece limitada. Decir que las causas y efectos del derecho son materia de estudio de la sociología jurídica es dejar de lado las reflexiones que desde otras disciplinas y sin la menor pretensión de alimentar a la sociología del derecho, se han producido en abundancia. Basta pensar, a manera de ejemplo, en un estudio sobre los efectos de una norma de contenido tributario (lo que podría ser tema de un estudio estrictamente económico) o uno sobre las causas y antecedentes de la Constitución colombiana desde una perspectiva meramente histórica. Naturalmente, podría argumentarse que en uno y otro caso, sin importar la ausencia de intención sociojurídica, ambos estudios contribuyen a enriquecer el ámbito de los estudios de la sociología del derecho, pero tal postura nos conduciría a afirmar que cualquier estudio en el que la vida social se vincule a normas jurídicas, es un estudio 
sociojurídico, lo cual nos obliga a preguntarnos, entonces, dentro del campo de las ciencias sociales, cuándo puede hacerse a un lado el efecto de las normas en las comunidades, cuando justamente su estructura normativa es lo que define buena parte de su existencia.

Como una forma para poder superar todas las sutiles complejidades al abordar una u otra definición, o para intentar una nueva, el académico colombiano Germán Palacio señaló:

“Los estudios sociojurídicos comprenden una acepción muy amplia: se trata de toda literatura que analiza la dimensión legal y normativa en relación con consideraciones o análisis que provienen de otras disciplinas sociales" (Palacio, 1996, 5). Más adelante señala "El campo socio-jurídico puede tener una acepción amplia, que es la que aquí hemos usado, y una definición más estrecha. En el sentido más amplio nos referimos a los estudios que vinculan el derecho con otros saberes sociales y humanos, incluida la economía, la política, la sociología, la criminología, la cultura, etc.

En un sentido estricto, lo socio-jurídico es el campo de la sociología del derecho. Esta disciplina trata de establecer las relaciones entre la normatividad jurídica positiva y las realidades sociales. El sistema de la dogmática jurídica no se interesa o no ofrece respuestas a los problemas de falta de aplicación o falta de eficacia de las normas" (Palacio, 1996: 20).

Dice Palacio en su texto "Las complejidades de la justicia: reflexiones sobre el Plan nacional de investigaciones sociojurídicas": "Al referirnos al objeto de la investigación socio-jurídica, lo hacemos en la acepción más amplia posible: la justicia no como código autocontenido, sino como realidad social, a la vez que como manera específica de acercarse al problema del orden social y al conjunto de problemas surgidos del orden mismo". Palacio aborda cuatro niveles analíticos de justicia: como un 'aparato codificado', un conjunto de estructuras, normas y reglas de juego que estructuran la vida social (justicia en cuanto institución); la justicia como concepto; la justicia como discurso y, por último, la justicia como pedagogía.

Sobresale en el análisis de Palacio el marco interdisciplinario que le otorga a la sociología del derecho, lo cual, sin lugar a dudas, es un elemento fundamental en los estudios sociojurídicos, Sin embargo, nos parece que esta aproximación, a pesar de los niveles analíticos propuestos, entraña un problema adicional, el cual es preciso evitar: el de referenciar como temática de la investigación sociojurídica a la justicia. Este concepto, más propio de la filosofía que de la sociología, nos remitiría a complejidades conceptuales ajenas al propósito de este texto.

Finalmente, Germán Silva, en un esfuerzo por integrar las perspectivas micro y macrosociológicas dentro de la sociología del derecho, la define como "la especialidad de la sociología que conoce acerca de las instituciones (estructuras) relativas al control jurídico en su relación con las prácticas sociales (interacciones) que acaecen en la sociedad" (Silva, 2002: 11). Esta conceptualización puede servir para acotar el objeto de la investigación sociojurídica, pues conforme a él, "la observación de las instituciones sociales, limitada a aquellas que guardan relación con el control jurídico, significa contemplar no solo los sistemas normativos establecidos, de manera formal o informal, o instituciones sociales estrechamente relacionadas con los operadores del derecho y la aplicación de las normas (...) (profesión jurídica, estructura judicial, ideologías profesionales, etc.), sino instituciones sociales de igual o mayor complejidad como la cultura, la economía, la organización estatal, la familia, etc., que según el caso y el aspecto involucrado, también 
repercuten sobre el control jurídico, mientras que las prácticas sociales, vistas en su relación con el control jurídico, implican no apenas advertir cómo se aplica el derecho en la realidad (lo que ocurre, muchas veces, de modo distinto a como aparece consignado en los estatutos legales, sino también examinar las actitudes y actuaciones de la población frente a los dispositivos de control" (Silva, 2002: 11). La extensión de la cita se justifica en la medida que articula el concepto anteriormente expuesto con los temas principales de la sociología del derecho.

Como se observa, son diversos los intentos por definir y clasificar a la sociología jurídica. Para algunos es simplemente un sector de la investigación sociológica caracterizado por la peculiaridad de su objeto; para otros, es el marco genérico de estudio de la interacción entre la sociedad y el derecho; otro grupo la circunscribe al ámbito de los análisis empíricos de actitudes o comportamientos jurídi$\cos ; y$, finalmente, para algunos académicos se extiende hasta abarcar cuanto atañe a las funciones del derecho, así como sus mecanismos de producción y aplicación ${ }^{3}$. Las anteriores definiciones poseen elementos comunes, los cuales, creemos, son las bases para definir a la sociología jurídica.

Cada una de las definiciones aquí presentadas tiene elementos de utilidad para fijar el alcance del concepto. Sin embargo, es claro que no hay unanimidad al respecto. Por otra parte, tampoco es posible construir una definición acumulativa, pues si bien no son contradictorias, en algunos aspectos la acumulación de definiciones puede forzar enunciados lógicamente incompatibles.

Resumiendo, la sociología jurídica:

1. Es vista o como una disciplina o como una especialidad de la sociología.
2. En general, no es definida como una disciplina del derecho.

3. En cuanto a sus métodos y forma de abordar el conocimiento, está más vinculada a la sociología que al derecho.

4. Aunque no de manera exclusiva, hace énfasis en el uso de métodos empíricos de investigación.

5. Pretende un análisis crítico de las situaciones jurídicas, una aproximación realista.

6. Se ocupa de los fines y las funciones del derecho, aunque no tiene la exclusividad sobre el tema.

Sin duda, son muchos los estudios que reúnen varios de los elementos que aquí se señalan. Dentro de ellos se destacan en la producción nacional los trabajos en torno a los conceptos de crimen, desviación, divergencia y control social, es decir, la criminología. En el apartado siguiente presentamos una visión general de la evolución de la criminología en el contexto nacional. Como podrá verse, el desarrollo de esta disciplina ha sido consistente y paradigmático en la evolución del pensamiento sociojurídico en América, razón por la cual le dedicamos una parte de este artículo. Como se verá, la correspondencia entre criminología y sociología no es total, ni es una constante. A pesar de ello, los avances logrados en la criminología son valiosos y merecen una mención especial en este artículo.

\section{La criminología y la sociología del derecho}

En estas líneas queremos resaltar el aporte de la criminología a la sociología del derecho, tema que ha generado importantes debates en la academia,

3 Cf. Antonio Enrique Pérez-Luño, prólogo de la versión en castellano de la “Introducción a la sociología” de Roger Coterrell (Coterrell, 1991: 7-11). 
que se preocupó por superar las generalidades y abstracciones del derecho entendido como norma jurídica y buscó una interpretación de lo jurídico a partir de características históricas y políticas concretas. Este análisis, que antecedió a la sociología jurídica, se hizo amparado en la construcción de un discurso propio, orientado a cuestionar las políticas de control social. La criminología tiene hoy en día un espacio propio en los programas de estudio del derecho.

\subsection{Ubicación de la criminología}

Dada la gran fuerza que ha tenido la producción criminológica en el país y su innegable influencia en el desarrollo de la sociología jurídica nacional, es necesario detenernos para ubicarlas en referencia mutua. Para este estudio, la vemos como una especie de la sociología del derecho que trata del tema penal, es decir como una sociología jurídica penal. No obstante, el asunto no ha sido pacífico en la teoría social, pues abundan las posiciones más disímiles sobre el problema. En el cuadro de la criminología, por ejemplo, para Lola Aniyar de Castro la criminología es una ciencia del control social en sus distintas variantes, entre ellas la jurídica no sólo penal (Aniyar, 1988); para Carlos Elbert la criminología es una disciplina, no una ciencia, con un carácter autónomo, por lo que descarta su identificación con la sociología (Elbert; 1996) mientras Roberto Bergalli (1988) y Alessandro Baratta (1986) coinciden con nuestra posición al describir la criminología como una especialidad de la sociología jurídica. Así mismo, en nuestro medio, sin ser dominante, la última postura tiene audiencia, por ejemplo en los casos de Mauricio Martínez y Germán Silva García. Por su parte, el asunto apare- ce más tranquilo entre los sociólogos del derecho, donde es más bien unánime la ubicación de la criminología como una parte de la sociología jurídica.

Desde luego, en la historia de la teoría criminológica han tenido cabida múltiples discursos que poco o nada tienen que ver con la sociología, sobre todo representados en las doctrinas de la biología, la antropología y la psicología, que adelantaron teorías sobre la criminalidad. La misma sociología criminal, en la actualidad en desuso, tenía que ver con la sociología general, pero seguía una línea de desarrollo del todo diferente y ajena a la sociología del derecho. En tales casos, sin duda, no podría establecerse una relación entre criminología y sociología jurídica. Pero la teoría contemporánea de la criminología, aún al ocuparse de la acción social, recurre de manera predominante al bagaje teórico y conceptual de la sociología del derecho para orientar su análisis, por lo que consideramos adecuada su ubicación en ese escenario ${ }^{4}$.

Ello no obsta para señalar que dentro del medio universitario nacional la criminología y la Sociología del Derecho han seguido caminos distintos, pocas veces coincidentes ${ }^{5}$. La criminología, bajo las expresiones de la biología, la antropología o la psicología, poseía una historia y una tradición diversa y, más o menos, independiente, donde aparecía unida en mayor grado a la medicina. Inclusive, como derivado de la autonomía relativa ya ganada, al involucrarse la sociología criminal o irrumpir después la Escuela de la Criminología Crítica en la subespecialidad, las sendas de desarrollo variado perseveraron. A ello pudieron haber contribuido algunas deficiencias teóricas. Por una parte, la pobreza sociológica de la sociología criminal, que en

4 Decimos de manera predominante, pues las crecientes necesidades de recurrir a conocimientos interdisciplinarios han llevado a muchos a hablar, incluso, de la necesidad de una teoría social considerada en términos generales.

5 No nos referimos a la cuestión epistemológica de su ubicación, asunto que a estas alturas ya consideramos superado, sino a los procesos políticos y administrativos relacionados con el desarrollo de la criminología, en todo caso subespecialidad de la sociología jurídica. Ella ha avanzado en forma preferentemente independiente respecto de la sociología del derecho. Por ejemplo, la criminología se sitúa, en el medio universitario, en los departamentos de derecho penal y criminología, la sociología del derecho aparece dispersa en varias unidades académicas o en un departamento propio, por lo general unida a la filosofía. 
vez de echar mano de la teoría sociológica, por regla general se reducía a la enunciación de una serie de factores sociales como determinantes de la criminalidad. De otra, el conocimiento superficial de la teoría sociológica dentro de la gran mayoría de los criminólogos críticos nacionales, abogados sin estudios de posgrado o pregrado en sociología, que conocían aspectos de la teoría sociológica por los resúmenes de algunos manuales de criminología y que, al ser dominante en el discurso de la criminología crítica, utilizaron un enfoque sobre todo político en sus trabajos.

Un efecto favorable para la sociología del derecho resultante de la aparente separación de la criminología contemporánea, es que la disciplina no se vio comprometida en la enconada y al final estéril disputa que los criminólogos sostuvieron con los dogmáticos del derecho penal en las décadas de 1970 y 1980, refriega que se había repetido y se sigue dando en muchos países de América Latina y Europa, por lo general con la derrota de los criminólogos.

\section{Otros temas relacionados}

La criminología no ha sido el único desarrollo importante. Existen otras aproximaciones que presentan potenciales vetas de desarrollo. Se trata de una serie de trabajos teóricos e investigaciones relacionadas con temas que también son abordados por el derecho y la sociología del derecho. Nos referimos a una extensa producción de la sociología, la economía, la filosofía social y la antropología nacional, que han tratado sobre la violencia política, la democracia, la organización territorial, el narcotráfico, la organización de la familia y los movimientos sociales. Esos trabajos, que la mayoría de las veces superan en calidad y cantidad a los provenientes de la sociología jurídica, en ocasiones hacen alusiones tangenciales al papel social o a la estructura del derecho, pero el epicentro de sus reflexiones no involucra lo jurídico, por tanto, al estar ausente la especificidad de lo jurídico como cuestión principal, son propios de otras especialidades no jurídicas de la sociología, o de la antropología o la economía y no se incluyen en este estudio, como tampoco las distintas acciones y procesos de organización que han dado lugar a su producción. Aun cuando por su calidad y porque versan sobre temas que constituyen objeto central de estudio de la sociología del derecho, son textos y fuentes de referencia indispensables para las labores de investigación y docencia sociojurídicas ${ }^{6}$.

También, bastante problemática resultaba la ubicación de los trabajos de sociología política. La sociología política, por cierto, es otra especialidad de la sociología general que trata de manera bastante próxima a la sociología jurídica algunos de los problemas regulados por el derecho. Así, cuestiones como el Estado, el poder, los sistemas de representación, las instituciones políticas, la organización de la administración, las relaciones internacionales, etc., no constituyen patrimonio exclusivo del derecho constitucional y la sociología jurídica; son examinadas también por la sociología política, aunque con muy poco énfasis en lo jurídico. En tal caso, con el mismo criterio, cuando el análisis sobre lo político no estaba conectado o no se refería al derecho o a instituciones político-jurídicas fue descartado. Por el contrario, aquellos trabajos de

$6 \quad$ Entre ellos cabría citar aquí: Ignacio Torres Giraldo (1974). Los inconformes, Bogotá, UNICCA, 4 tomos. Alejandro Angulo y otros (1980). Derechos humanos... ¿ ¿Derechos del pueblo? Bogotá, CINEP. Gonzalo Sánchez; Donny Meertens (1983). Bandoleros, gamonales y campesinos, Bogotá, El Ancora. AA.W. (1985). Once ensayos sobre la violencia, Bogotá, CEREC y Centro Gaitán. Eduardo Fonseca Galán (1987). Los combatientes del Ilano 1949-1953, Bogotá, Universidad INCCA de Colombia. William Ramírez Tobón (1990). Estado, violencia y democracia, Bogotá, Tercer Mundo e IEPRI. Ciro Krauthausen y Luis Fernando Sarmiento (1991). Cocaína \& co., Bogotá, Tercer Mundo e IEPRI. Francisco Thoumi (1994). Economía política y narcotráfico, Bogotá, Tercer Mundo. Alonso Salazar y otros (1996). La génesis de los invisibles, Bogotá, Programa por la Paz de la Compañía de Jesús. William Ramírez Tobón (1997). Urabá. Los inciertos confines de una crisis, Bogotá, Planeta. Alfredo Rangel Suárez (1998). Colombia: guerra en el fin de siglo, Bogotá, Tercer Mundo y Universidad de Los Andes. Jesús Antonio Bejarano Ávila y otros (1998). Colombia: inseguridad, violencia y desempeño económico en las áreas rurales, Bogotá, FONADE y Universidad Externado de Colombia. 
sociología político-jurídica involucrados con el llamado derecho público, fueron considerados como una de las vertientes más importantes de la actividad sociojurídica?

Finalmente, la antropología jurídica es una subespecialidad de la antropología social, que no pertenece al mundo de la sociología. Empero, en la actualidad distinguir entre la antropología social, la psicología social y la sociología es una tarea imposible. Por tanto, aunque nos ubiquemos en un campo más general que algunos gustarían llamar de la teoría social de lo jurídico -no de la sociología del derecho-, deberíamos incluir en este campo los trabajos de antropología jurídica. Todo lo cual no implica que prediquemos una desaparición de esas disciplinas, pues existen otras subespecialidades que no tienen una relación mayor con la sociología, como la antropología física, la paleontología o la psicología clínica, además de una tradición en el saber científico imposible de negar y enfoques, énfasis y objetos de conocimiento no siempre comunes.

\section{Conclusión}

La sociología jurídica es una disciplina de reciente formación, por ello su objeto de trabajo aún sigue siendo un elemento de discusión por académicos de diversas latitudes. Hoy, frente a su objeto y método de estudio, no existe unanimidad por parte de la comunidad académica, sin embargo, se presentan algunos elementos que pueden caracterizar el campo de estudio de la sociología jurídica, estos elementos son: en primer lugar, la sociología jurídica es ubicada como una especialidad de la sociología; en segundo lugar, los métodos y las formas de estudio están vinculados con la sociología; en tercer lugar, la mayoría de los sociólogos jurídicos hacen énfasis en el uso de métodos em- píricos de investigación; en cuarto lugar, la sociología del derecho pretende un análisis crítico de las situaciones jurídicas frente a la realidad; por último, la sociología jurídica se ocupa preferentemente de los fines y las funciones del derecho.

Adicionalmente, la sociología jurídica ha estado en permanente contacto con otras disciplinas de las ciencias sociales, su vínculo se da a través de numerosos estudios que son abordados de igual manera por la sociología del derecho y por economistas, politólogos, antropólogos y sociólogos. El involucrarse en temas como la violencia política, la democracia, el narcotráfico, los ordenamientos jurídicos culturales, etc., obliga a los sociólogos del derecho a desarrollar diálogos interdisciplinarios.

\section{Bibliografía}

COTTERRELL, Roger (1991). Introducción a la sociología del derecho. Ariel Derecho, Barcelona, España.

CORREAS, Oscar (1999). La sociología jurídica. Fontana, México D.F., México.

DE LA TORRE, Jesús Antonio (1997). Sociología jurídica y uso alternativo del derecho. México, Instituto de Cultura de Aguas Calientes.

PALACIO, Germán (1993). Pluralismo jurídico. Universidad Nacional de Colombia, Facultad de Derecho y Ciencias Políticas y Sociales, Bogotá, Colombia.

PALACIO, Germán (1996). La investigación sociojurídica: Para desafiar la estéril autocomplacencia profesional. En: Revista Pensamiento Jurídico No. 6, Universidad Nacional de Colombia, Bogotá, Colombia.

7 Ejemplos de socilogía política conectados con el derecho lo constituyen los trabajos de Francisco Leal Buitrago: “Política e intervención militar en Colombia" (1973); "Análisis histórico del desarrollo político nacional 1930-1970" (1973); "Estado y política en Colombia" (1984), y de Álvaro Echeverri: "El poder y los militares" (1978); "Elites y proceso político en Colombia" (1997) 
REHBINDER, Manfred (1981). Sociología del derecho. Editorial Pirámide, Madrid, España.

ARDILA, Édgar (2002). “Pluralismo jurídico apuntes para el debate".En: El Otro Derecho, \# 26-27, ILSA, Bogotá, Colombia.

SILVA, Germán (2002). "El proceso de la investigación sociojurídica en Colombia”. En: Revista Diálogos de Saberes No. 15, abril - junio, Universidad Libre, Bogotá, Colombia.

CARBONIER, Jean (1982) Sociología jurídica. Editorial Tecnos, Madrid, España.

SANTOS, Boaventura De Sousa (1991). Estado, derecho y luchas sociales. ILSA, Bogotá, Colombia.

SORIANO, Ramón (1997). Sociología del derecho. Editorial Ariel, Barcelona, España.

BURGOS, Germán (1993). "Las facultades de derecho salen a la calle". En: El Otro Derecho, \# 13, ILSA, Bogotá, Colombia.

ARENAS, Carlos y GÓMEZ, Gabriel (2000). “En busca de justicia en los tiempos de reformas judiciales: estudios de caso en Colombia, Perú y Venezuela". En: El Otro Derecho, \# 25, ILSA, Bogotá, Colombia.
RODRÍGUEZ, César (2000). “Globalización reforma judicial y Estado de derecho: El regreso de los programas de derecho y desarrollo" En: El Otro Derecho, \# 25, ILSA, Bogotá, Colombia.

TREVES, Renato (1988). La sociología del derecho. Orígenes, investigaciones, problemas. Editorial Ariel, Barcelona, Epaña.

ANIYAR de Castro, Lola (1988). “Un debate sin punto final". En: Doctrina penal. Buenos Aires, Depalma.

ELBERT, Carlos (1996). Criminología latinoamericana. Buenos Aires, Argentina.

BARATTA, Alessandro (1986). Criminología crítica y crítica del derecho penal. México, Siglo Veintiuno, traducción de Álvaro Búnster, pp. 14 y 15.

BERGALLI, Roberto (1988). "El control penal en el marco de la sociología jurídica". En: Doctrina penal. Buenos Aires, Depalma, pp. 583 y ss.

GURVITCH, Georges (1945) Sociología del derecho. Editorial Rosario, Argentina. Traducción de Ángela Romera Vera.

ROBLES, Gregorio (1997). Sociología del derecho. Editorial Civitas, Madrid, España. 